Spat i al resol ved hi gh- energy parti cle di agnost i c system usi ng ti me- of- $\mathrm{fl}$ i ght neut ral parti cle anal yzer in Large Hel i cal Devi ce

\begin{tabular}{|c|c|}
\hline $\begin{array}{l}\text { jour nal or } \\
\text { publ i cat } i \text { on titl e }\end{array}$ & Revi ew of Sci ent i f i c I nst r ument s \\
\hline vol une & Vol . 74 \\
\hline nunber & No. 3 \\
\hline page $r$ ange & pp. 18781882 \\
\hline year & 2003-03-01 \\
\hline URL & ht t p: //hdl . handl e. net /10655/3268 \\
\hline
\end{tabular}




\title{
Spatial resolved high-energy particle diagnostic system using time-of-flight neutral particle analyzer in Large Helical Device
}

\author{
Tetsuo Ozaki ${ }^{\mathrm{a})}$ and Sadayoshi Murakami \\ National Institute for Fusion Science, Toki, Gifu 509-5292, Japan \\ Pavel Goncharov \\ Graduate University for Advanced Studies, Hayama, Kanagawa 240-0193, Japan
}

Tsuguhiro Watanabe, Shigeru Sudo, Kazumichi Narihara, Kenji Tanaka, Shin Kubo, Takashi Simozuma, Hiroshi Idei, Yoshihide Oka, Masaki Osakabe, Yasuhiko Takeiri, Katsuyoshi Tsumori, Katsumi Ida, Mamoru Shoji, Hideya Nakanishi, Mamiko Sasao, Osamu Kaneko, Kazuo Kawahata, Yukio Nakamura, Nobuyoshi Ohyabu, Osamu Motojima, and the G1/G2 Experimental Group

National Institute for Fusion Science, Toki, Gifu 509-5292, Japan Graduate and University for Advanced Studies, Hayama, Kanagawa 240-0193, Japan

(Presented on 9 July 2002)

\begin{abstract}
The time-of-flight-type neutral particle analyzer has an ability of horizontal scanning from 40 to $100^{\circ}$ of the pitch angle. The information from the spatially resolved energy spectrum gives not only the ion temperature but also the information of the particle confinement and the electric field in plasmas. We have been studying the energy distributions at various magnetic configurations in the neutral beam injection (NBI) plasma. The spatially resolved energy spectra can be observed during long discharges of the NBI plasma by continuous scanning of the neutral particle analyzer. The shape of spectra is almost similar from $44^{\circ}$ to $53^{\circ}$. However, the spectra from $55^{\circ}$ are strongly varied. They reflect the injection pitch angle of the beam. The pitch angle scanning experiment during the long discharge of NBI plasma has also been made under the reversal of the magnetic field direction. NBI2 becomes counter injected with the reversal. We can easily observe the difference between co- and counter injections of NBI. During the electron cyclotron heating in the low-density plasma for the formation of the internal thermal barrier, large neutral particle increase or decease can be observed. The degree of the increase/decrease depends on the energy and the density. The reason for the variation of the particle flux is that the orbit of the trapped particle changes due to the electric field formed by the strong electron cyclotron heating. (C) 2003 American Institute of Physics.
\end{abstract}

[DOI: $10.1063 / 1.1537883$ ]

\section{INTRODUCTION}

It is important to observe the energy dependence of the pitch-angle distribution to investigate the confinement of high-energy particles. For this purpose the neutral particle analyzer should have a wide observation energy range above the maximum energy of neutral beam injection (NBI) (or of particle accelerated by ion cyclotron resonance heating) and an ability to separate the particle species of hydrogen, deuterium, and helium -3/-4. It is important that it has the ability to exclude the noise from D-D neutrons and the strong radiation during heating. We have been improving the time-of-flight (TOF) neutral particle analyzer (NPA) for the Large Helical Device (LHD), which had been developed in ENEA Frascati. ${ }^{1}$ The control and data acquisition systems are designed to be suitable for long discharges in LHD. The horizontally movable stage is prepared to investigate the pitch angle distribution of the high-energy particles in plasma. Here, we demonstrate two typical experimental results using the instrument.

${ }^{a)}$ Electronic mail: ozaki@nifs.ac.jp
In helical devices, the particle orbit of the co-injection beam with respect to the direction of the toroidal magnetic field is different from that of the counter injection. According to the calculation, the guiding center of the particle orbit is close to the magnetic axis and shifted to the high magnetic field side in the co- and counter injection, respectively. The tendency is remarkable in the low magnetic field. On the contrary, that of the counter injection is localized near the high magnetic field side. Therefore, some of the particles are lost through collision with the inner wall. In the fourth experimental campaign, the neutral beam injection (NBI) 1 as counter injection and NBI 2 as co-injection are prepared. In the fifth cycle, NBI 3 was added as a counter-injection beam. NBI 1 and 3 change to the co-injection and 2 changes to the counter injection by the reversal of the magnetic field direction in some shots in the fifth cycle. The comparison between the co- and counter injection is mentioned in Sec. III.

Another experiment is performed by the highly localized electron cyclotron resonance heating $(\mathrm{ECH})$ during the low density NBI plasma. In this condition, the thermal transport barriers in plasma can be expected. The presence of the barrier becomes a mandatory condition to attain high perfor- 


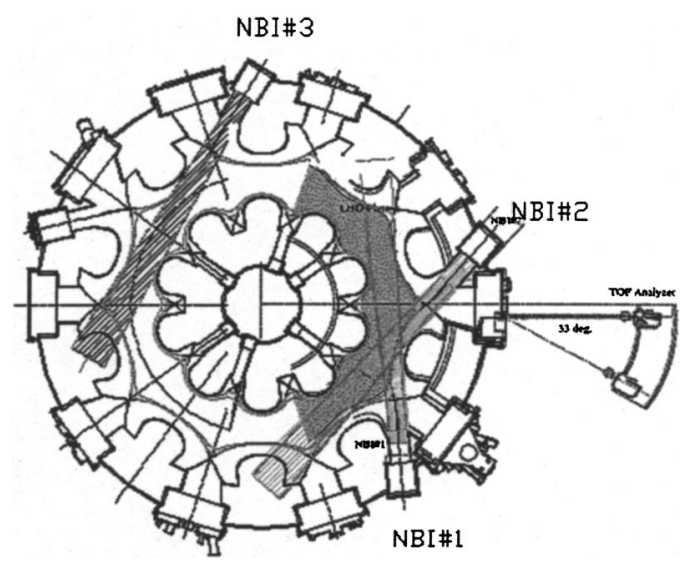

FIG. 1. The experimental configuration of LHD, NBI, and NPA. The analyzer can be scanned from $-2^{\circ}$ to $33^{\circ}$.

mance plasma with the high electron temperature and improved energy confinement. The transition of the radial electric field from negative to positive in the plasma is strongly related to the barrier formation. It is very interesting for us that the neutral particle flux originating from trapped particles increases or decreases by the transition of the electric field. The reason is that it is evidence of the transition of the potential in plasma and that the neutral particle may have physical information near the barrier. The dependence of the variation of the neutral particle flux on the density and the electric field is described in Sec. IV.

\section{DIAGNOSTICS AND EXPERIMENTAL SETUP}

The time-of-flight-type neutral particle analyzer (maximum/minimum observable energy $0.5 / 370 \mathrm{keV}$, typical energy resolution $7 \%$ ) has the capability of a high signal to noise $(\mathrm{S} / \mathrm{N})$ ratio against the radiation including soft/hard $\mathrm{x}$ rays from plasmas. The high $\mathrm{S} / \mathrm{N}$ ratio is obtained by using the anti-coincidence method of the time of flight. The NPA has 16 energy channels, three different mass separations, and a high mass rejection of $1 / 1000$. The main specifications of the NPA itself are described in Ref. 2. The scanning speed increases three times more than the previous system. It measures the scanning of the energy spectra during long discharge. The analyzer on the movable stage is set at the equatorial plane of LHD on the 10-O port. There are two NBIs (NBI 1 and 2) at neighboring ports to 10-O (Fig. 1). NBI 3 is installed in the parallel direction with NBI 2 from the fifth campaign. In particular, the beam path of NBI 1 crosses the sight line of the analyzer. Therefore a large amount of particles from the plasma center can be expected, which is suitable for the high-energy distribution measurement.

The outputs of the detectors enter programmable time differential analyzers with amplifiers (N209A, CAEN Co.). N209A amplifies the TOF detector start and stop signals, and produces a NIM pulse when both signals are within a programmed delay and time gate. NIM pulses are transferred to CAMAC latching scalars (C-TS308, Technoland Co.). The latching scalar counts NIM pulses every $5 \mathrm{~ms}$ and stores them in the buffer memories. After the discharge, all data are locally saved in the file of a WINDOWS NT machine. The
PV-WAVE application (Visual Numerics Co.) on WINDOWS NT is used as the data acquisition and the device control system.

The horizontal scanning is performed with the pivot in front of the $10-\mathrm{O}$ port by using a motor, which is remotely controlled from $-2^{\circ}$ to $+31^{\circ}(0$ degrees indicates the direction perpendicular to the $10-\mathrm{O}$ port flange surface). The angles are equal to the pitch angle (the angle between the magnetic axis and the sight line) of $100^{\circ}$ and $40^{\circ}$, respectively. The scanning speed of the stage is $0.17^{\circ} / \mathrm{s}$, which has sufficient ability to study the pitch angle distribution during the long discharge. The position (=angle) can be measured from the length of the stainless wire between the stage and a fixed point. The position is always monitored by a charge coupled device camera from the control room. At the same time, the position data are sent to the WINDOWS computer during the long discharge. The timing data can be provided to the computer from the timer, which is triggered by the LHD discharge initiation.

The ECH system consists of two 82.7 and two $84 \mathrm{GHz}$ gyrotrons for fundamental resonance heating, and three 168 $\mathrm{GHz}$ gyrotrons for second harmonic heating at $3 \mathrm{~T}$. The electron temperature profile in the equatorial plane, and the electron line density (or profile) are measured by Thomson scattering and an interferometer, respectively.

\section{COMPARISON BETWEEN CO- AND COUNTER NBIs}

To investigate the high-energy particle confinement, the pitch angle scan experiment during long discharges is performed. We choose scanning during the long discharge rather than the shot by shot in order to obtain a precise result. The accurate comparison between co- and counter injection can be expected so the horizontal scan of the analyzer during long discharge is done both in the normal and reversed magnetic fields. The spatially resolved energy spectra can be observed during the long discharge of NBI plasma by continuously scanning the neutral particle analyzer., ${ }^{3,4}$ Figure 2(a) shows the time evaluated (=angular distributed) threedimensional spectrum obtained by overlapping three similar NBI plasma discharges. The pitch angle means the angle between the sight line and the central magnetic axis. In these discharges, the plasmas are initiated by the ECH heating, after that NBI 2 (co-injection) sustains the plasma for 40-60 s. The scanned pitch angle is from 44 to $74^{\circ}$. The electron density measured by the interferometer is kept at almost 2 $\times 10^{19} \mathrm{~cm}^{-3}$ during the discharge except during the plasma initiation phase. At the initiation phase by $\mathrm{ECH}$, the highenergy particle flux is enhanced as the density is too low and the background neutral density is high. The injected hydrogen neutral beam energy of NBI 2 is only $130 \mathrm{keV}$ because the original ion source polarity is negative. To make the pitch angle dependence clear, the contour plot of Fig. 2(a) is shown in Fig. 2(b). The shape of the spectra is almost similar from $44^{\circ}$ to $53^{\circ}$. However, the spectra from $55^{\circ}$ are strongly varied. They reflect the injection pitch angle of the beam according to the simulation. The beam maintains the pitch angle at incidence until the beam energy reaches the energy 


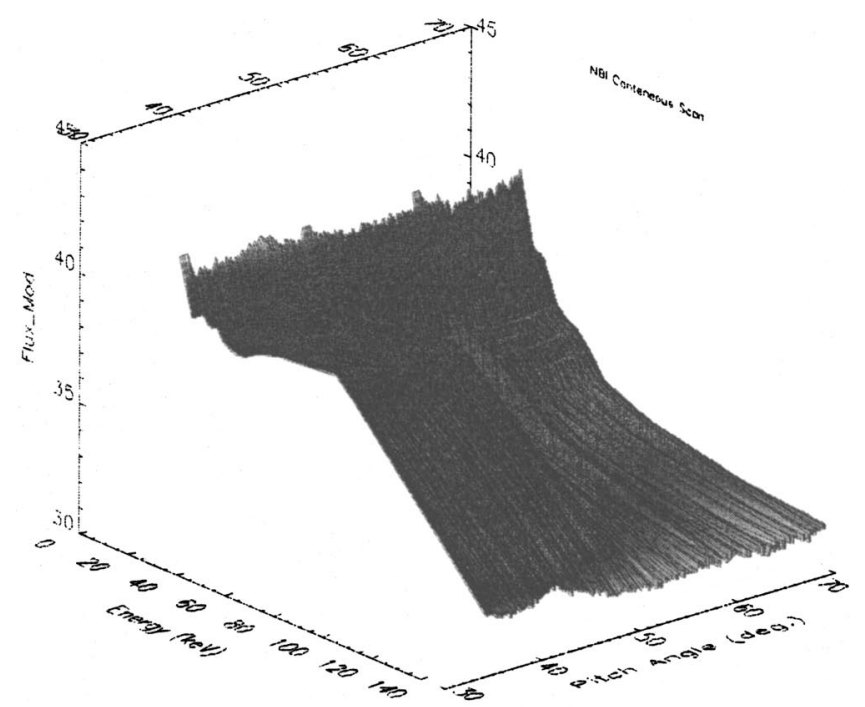

(a)

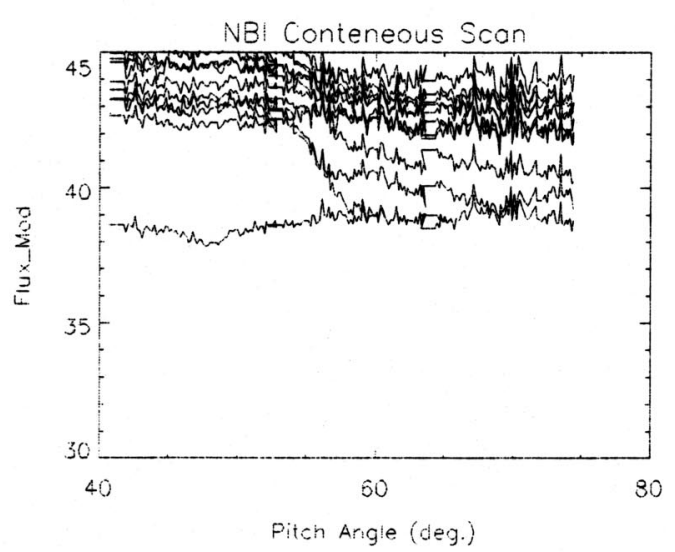

(b)

FIG. 2. (a) The spatially resolved neutral particle energy spectrum in NBI long discharge (co-injection). (b) The contour plot of (a).

at which the pitch angle scattering occurs by the energy loss due to the electron collision. The low flux region can be observed around $10-15 \mathrm{keV}$, which is 15 times the electron temperature. The energy region may be equal to the energy at which the pitch angle scattering occurs.

The pitch angle distribution and the contour plot are shown in Figs. 3(a) and 3(b), respectively, when the magnetic field is reversed. The large variation at $48^{\circ}$ can be observed. The pitch angle of the particle is almost conserved in the high-energy region because the pitch angle scattering occurs near an energy of 15 times of the electron temperature. It is unbelievable that only the particles with large pitch angle are localized near the plasma center where the background neutrals are not abundant. Therefore it is reasonable that the particle with the pitch angle between $48^{\circ}$ and $53^{\circ}$ is lost. The neutral particle flux in the counter injection is less than that in the co-injection as in the modulation experiment. ${ }^{6}$ The main reason is that the guiding center of the particle orbit in the counter injection is shifted to the high field side. Another candidate for the flux decrease comes from the particle loss with large pitch angle. However, the loss of the counter injected particle does not strongly affect to the heating efficiency and the stored energy because the

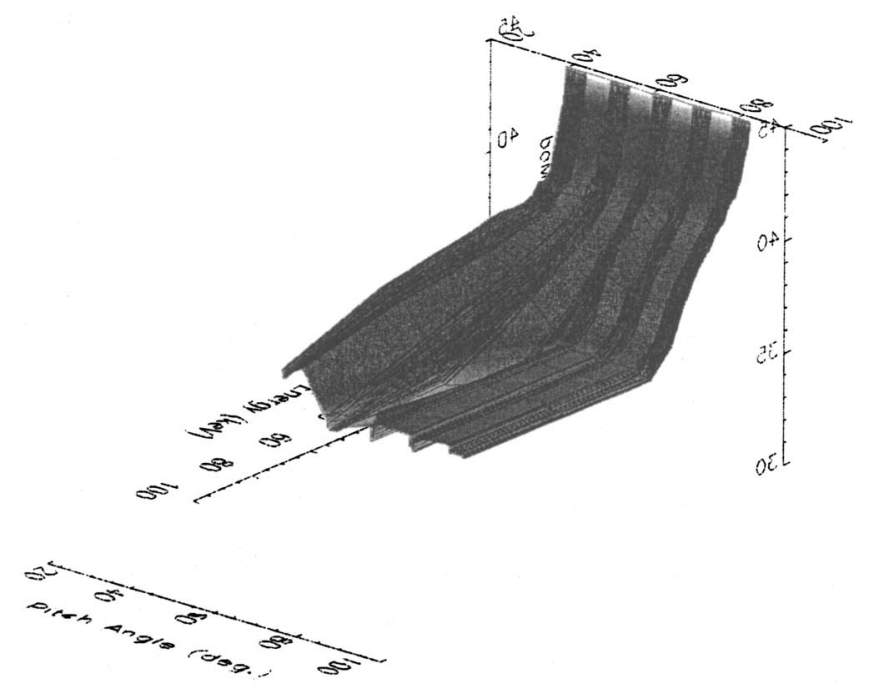

(a)

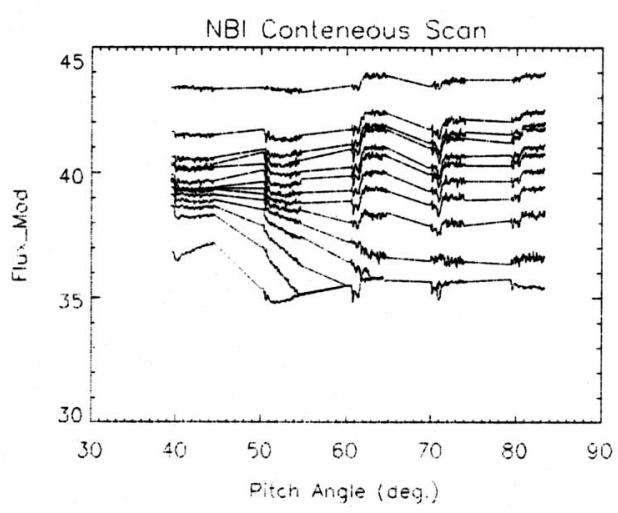

(b)

FIG. 3. (a) The spatially resolved neutral particle energy spectrum in NBI long discharge (counter injection). (b) The contour plot of (a).

contribution of the particle with large pitch angle is not so great. ${ }^{5,6}$

\section{NEUTRAL PARTICLE FLUX IN THE ITB FORMATION}

In the internal transport barrier (ITB) experiment, ${ }^{7}$ the highly intense ECH (230-530 kW) is applied from 1.2 to 2.3 $\mathrm{s}$ in the low density NBI plasma less than $1.3 \times 10^{19} \mathrm{~m}^{-3}$. Not only NBI 1 but also NBI 2 and/or 3 are applied at some shots in the experiment. Typical wave forms are shown in Fig. 4. Some different plasma densities and different ECH powers are also tried. The NPA is fixed at the pitch angle of $70^{\circ}$ on the equatorial plane. If only NBI 1 , which is the counter beam against the magnetic field direction, is applied, the trapped particles can be mainly observed at this position. When the highly localized ECH is applied to the low density NBI plasma, the formation of the electron internal transport barrier can be observed. The transition of the radial electric field from negative to positive in the plasma is strongly related to the barrier formation. At the same time, the neutral particle flux increases in low- and high-energy regions and decreases in the middle energy region as shown in Fig. 5(a). The electron temperature measured by the Thomson scattering near the plasma center increases up to twice or more 


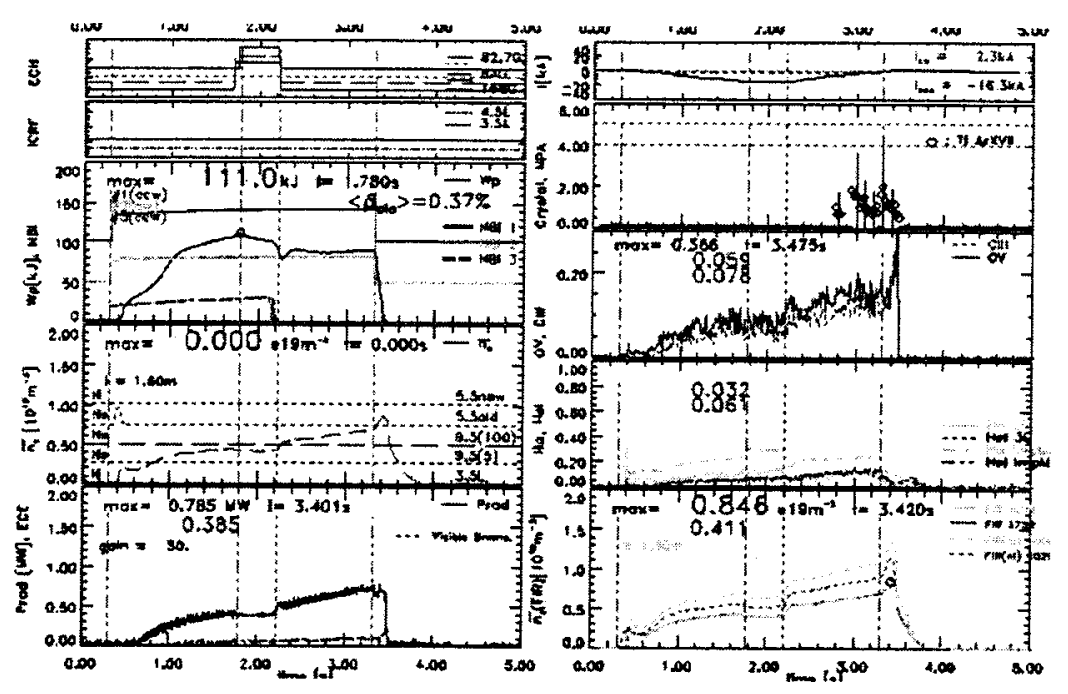

FIG. 4. Typical wave forms in the ITB experiment.

[Fig. 5(b)]. The density profile during ECH application does not vary so much from that before the ECH application. Figure 6(a) shows the density dependence of the neutral particle flux deviation between with and without ECH application divided by "without ECH." The value is $(B-A) / A$, where $A$

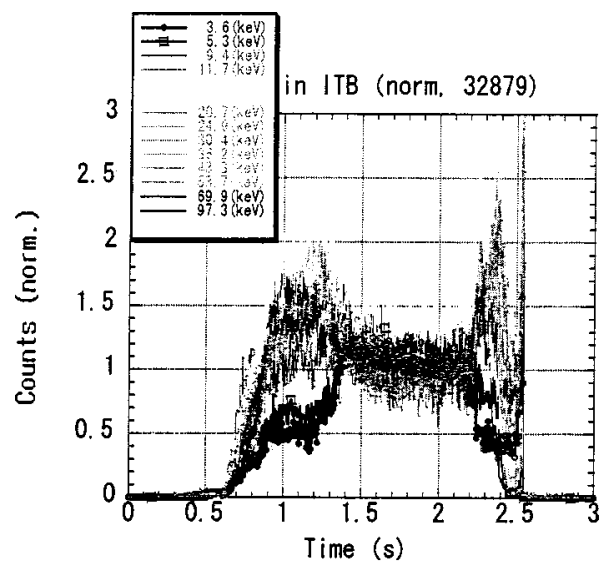

(a)

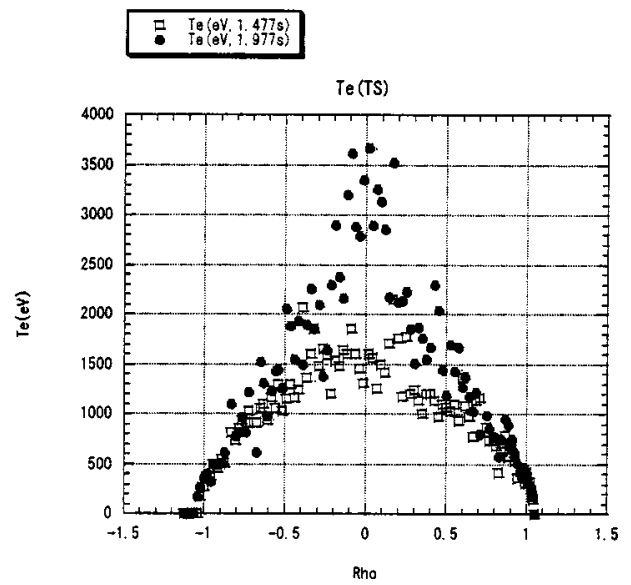

(b)

FIG. 5. (a) The normalized neutral particle flux at each particle energy. The $\mathrm{ECH}$ is applied from 1.2 to $2.3 \mathrm{~s}$. The flux at the low-energy region increases but that at the high-energy region decreases. (b) The electron temperature profile with/without ECH application. The central electron temperature is strongly enhanced. and $B$ are the flux without and with $\mathrm{ECH}$, respectively. The flux deviation depends inversely on the plasma density. At the low density, the variation of the electron temperature $[=(C-D) / C$, where $C$ and $D$ are the temperature without and with $\mathrm{ECH}$, respectively] is large. Figure 6(b) indicates the temperature variation dependence of the neutral particle flux. The flux deviation is large when the temperature variation is large. The increase of the temperature during the $\mathrm{ECH}$ phase indicates the strong formation of the internal transport barrier near rho $=0.5$. The formation of the barrier is related to the transition of the electric field in the plasma. According to the potential measurement using the charge exchange recombination spectroscopy, the electric field near the plasma

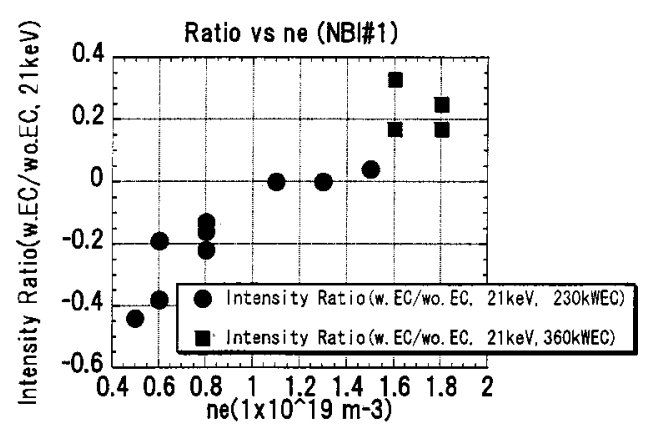

(a)

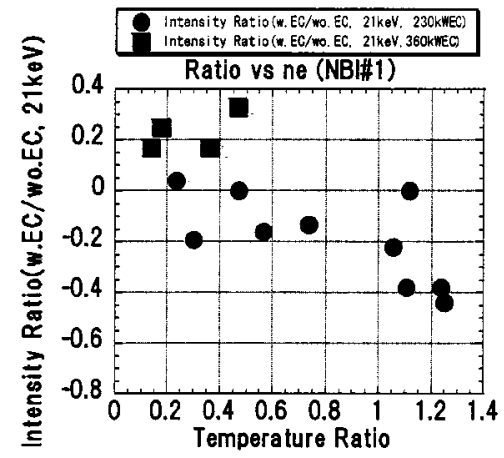

(b)

FIG. 6. (a). The neutral particle flux increase with/without ECH vs the plasma electron density. (b) The neutral particle flux increase with/without $\mathrm{ECH}$ vs the variation of the central electron temperature. 


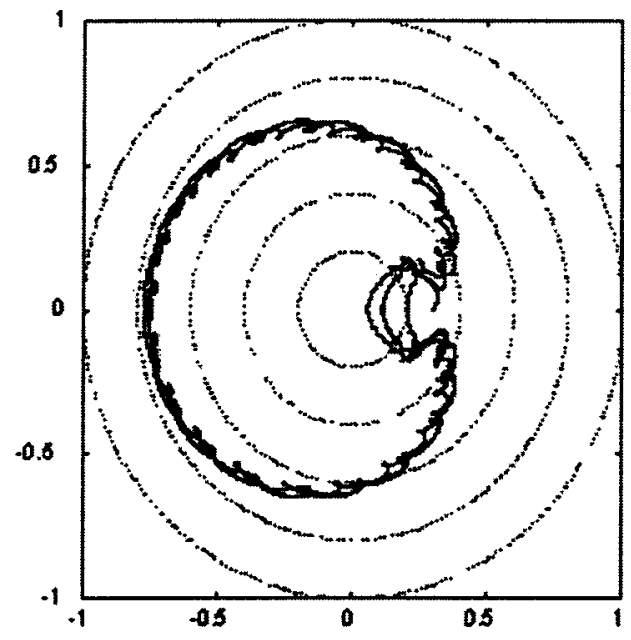

(a)

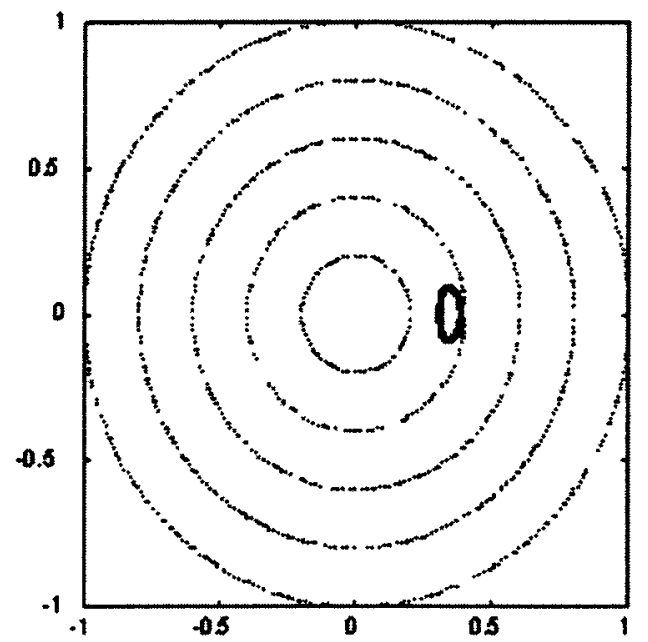

(b)

FIG. 7. (a) Particle orbit without ECH in simulation GNET. (b) Particle orbit with ECH in simulation GNET.

center changes to positive during ECH application although it is negative before ECH application in the low electron density. Especially the variation of the electric field within rho $=0.5$ is too large. The deviation of the neutral particle flux comes from that of the electric field within rho $=0.5$. The orbit of the trapped particle changes due to the variation of the electric field. The degree of the change of the orbit is correlated with the strength of the electric field.

When the NBI 2 (=co-injection) is additionally applied, the behavior of the particle orbit becomes complicated. At this NPA position, the passing particle from NBI 2 and the trapped particle can be observed at the same time. The varia- tion of the neutral particle flux of the density and temperature deviation is not stronger than that in only the NBI 1 case.

The orbit of the trapped particle is simulated using the GNET code as shown in Figs. 7(a) and 7(b). The test particle is the energy of $20 \mathrm{keV}$, the start point at $\mathrm{rho}=0.3$, and the pitch angle of $80^{\circ}$. Figures 7 (a) and 7(b) show the projections of the particle orbits on the poloidal cross section of the plasma without and with ECH, respectively. The radial electric field with the ECH phase, which is experimentally obtained from charge exchange spectroscopy, is different from that without ECH. The orbit with ECH is obviously different from that without ECH. The variation of the neutral particle flux with and without ECH is caused by the difference of the potential profiles.

\section{CONCLUSION}

The time-of-flight-type neutral particle analyzer, which is able of horizontal continuous scanning during long discharges, can be operated in LHD. The scanning speed increases three times more than the previous system. It measures the scanning of the energy spectra during long discharge. Two typical experiments demonstrate the best use of this diagnostic tool. The experiments for the comparison between the co- and counter neutral beam injection are performed. The pitch angle distribution of the neutral particle spectrum in both injections can be precisely obtained by scanning the analyzer in long discharge changing and by the magnetic field direction. In the counter injection the neutral particle flux is not so much because the particle orbit passes through the low background neutral region. It can be found that the particle with the large pitch angle is lost in the counter injection from the spectrum of the pitch angle distribution of the neutral particles. The large variation of the neutral particle flux can be observed during ITB formation phase. It is strongly related to the change of the trapped particle orbit by the internal electric field.

\section{ACKNOWLEDGMENTS}

The authors thank the Diagnostic technology group for tuning the analyzer and Director General Professor Fujiwara for his encouragement.

${ }^{1}$ G. Bracco, G. Betello, S. Mantovani, A. Moleti, B. Tilia, and V. Zanza, Rev. Sci. Instrum. 63, 5685 (1992).

${ }^{2}$ T. Ozaki et al., Rev. Sci. Instrum. 71, 2698 (2000).

${ }^{3}$ T. Ozaki et al., Plasma Phys. Controlled Nucl. Fusion Res. 3, 444 (2000).

${ }^{4}$ K. Saito et al., Plasma Phys. Controlled Fusion 44, 103 (2002).

${ }^{5} \mathrm{~T}$. Watanabe, http://www.lhd.nifs.ac.jp/lhdorb.html

${ }^{6}$ T. T. Ozaki et al., Proc. of the 29th EPS, P-4.065 (2002).

${ }^{7}$ T. Shimozuma et al., Phys. Rev. Lett. (unpublished). 